\title{
Factors associated with influenza vaccine coverage among patients with diabetes: Korea National Health and Nutrition Examination Survey 2016-2018
}

\author{
A. Lum $\operatorname{Han}^{1}$ (D) \\ Received: 17 November 2020 / Accepted: 4 July 2021 / Published online: 27 July 2021 \\ (c) Research Society for Study of Diabetes in India 2021
}

\begin{abstract}
Background Although the influenza vaccine has been proven to be effective, this common disease has high morbidity and mortality rates. Moreover, adults with diabetes are at a high risk of influenza-mediated morbidity and mortality.

Purpose of the study With the increasing prevalence of diabetes, influenza is more lethal in diabetics; thus, we aimed to investigate the factors associated with influenza vaccination coverage in patients with diabetes.

Methods Cross-sectional data were obtained from the Korea National Health and Nutrition Examination Survey (20162018). We retrospectively analyzed whether sociodemographic, health-related, and medical factors are associated with influenza vaccination coverage in patients with diabetes. We performed a complex sample logistic regression analysis and estimated the odds ratios (ORs) by adjusting for statistically significant factors.

Results The number of survey subjects was 18,553 . The vaccine coverage rate among patients with diabetes was $60.6 \%$. In the univariate analysis, sex, educational level, smoking, exercise, drinking, marital status, private health insurance, activity limit, economic activity, age, and EuroQol-5 Dimension scores, which were used to assess health-related quality of life, were associated with vaccination coverage. In the multivariate analysis, only age and economic activity were associated with vaccination coverage. The vaccination coverage rate was higher for people who did not undertake economic activities and who were older (OR 1.512 (1.087-2.105), OR 2.212 (1.822-2.686), respectively, $p<0.001$ ).

Conclusion National interventions involving public health centers are necessary to encourage influenza immunization for patients with diabetes, especially younger patients and those who work or undertake economic activities.
\end{abstract}

Keywords Diabetes $\cdot$ Influenza vaccination coverage $\cdot$ South Korea

\section{Introduction}

Despite the proven effectiveness of the influenza vaccine, this common disease still has high mortality rates [1]. Vaccination is the most cost-effective way of reducing the risk of influenza and its complications, especially in older adults and patients with chronic diseases [1-3]. Seasonal influenza pandemics are disastrous for public health, especially during winter [1]. Further, adults with diabetes are at a high risk of influenza-mediated morbidity and mortality $[4,5]$.

A. Lum Han

qibosarang@naver.com

1 Department of Family Medicine, Wonkwang University Hospital, Sinyong-dong 344-2, Iksan, Jeollabuk-do 54538, South Korea
A systematic review of 15 studies published between January 2000 and March 2017 [6], provided data on the immunogenicity, safety, effectiveness, and cost effectiveness of influenza vaccination in diabetics. The immunogenicity of influenza vaccination in diabetic patients after vaccination is comparable to that of healthy participants. Vaccination against influenza is reported to reduce the risk of hospitalization and fatality in diabetics, especially those over the age of 65 years. As such, the need for and value of annual influenza vaccination for diabetics to alleviate serious complications, such as hospitalization and death, is high. In another study, data from the Health and Nutrition Examination Survey III-VI (2005-2015) were analyzed to identify the factors associated with influenza vaccine coverage among diabetic patients in Korea [7]. The study found that socioeconomic and health-related factors are associated with influenza vaccination among diabetic patients. 
In a Canadian study, patients with diabetes, even those aged under 65 years, were found to be susceptible to influenza and had higher rates of influenza-attributable all-cause hospitalization [4]. The reason for the increased vulnerability to influenza in patients with diabetes is their impaired immune response; they have abnormal CD4/CD8 ratios, and their natural killer cells and monocytes do not function well $[8,9]$.

For effective prevention against influenza, the World Health Organization (WHO) recommends a vaccination coverage rate (VCR) of at least $75 \%$ in vulnerable populations [10]. However, recent data demonstrate that the recommended influenza VCR is not being achieved in most countries [11-13].

In 2011, 366 million patients worldwide were recorded as having diabetes, and according to the International Diabetes Federation, this number is expected to increase by 552 million by 2030 [14]. As the prevalence of diabetes increases and people with diabetes remain vulnerable to infection $[15,16]$, it is important to examine the influenza VCR in this population. In South Korea, national support for the influenza VCR is very high, and it contributes to various indicators, including influenza-related outpatient consultations, hospitalization and excess mortality, qualityadjusted life years, and the incremental cost-effectiveness ratio [17].

While several studies on the influenza VCR have been conducted in South Korea, these have focused only on chronic obstructive pulmonary disease [18], other chronic diseases [19, 20], or older adults [21-23]. Thus, the aim of this analysis was to evaluate the socioeconomic, personal, and environmental factors associated with the influenza VCR among people with diabetes, who are very vulnerable to infection.

\section{Materials and methods}

\section{Data source}

The present analysis was conducted using cross-sectional data obtained from the 2016-2018 Korea National Health and Nutrition Examination Survey (KNHANES). The KNHANES is annually conducted to assess the health and nutritional status of the non-institutionalized civilian population in South Korea. These investigations, which include a health interview survey, a health examination survey, and a nutrition survey, are conducted by trained investigators. In the present analysis, a clustered and stratified random sampling method based on national census data was used. All factors included in the analysis were assessed using the KNHANES methodology to ensure objectivity of data.
Participants below the age of 19 years, as well as those whose survey items were missing, were excluded from the analysis.

Further, diabetes was the only disease included in the survey; all other chronic diseases, such as cardiovascular disease, respiratory disease, liver disease, and cancer, were excluded.

\section{Ethical considerations}

The analysis was approved by the Clinical Trial Screening Committee of $\mathrm{W}$ Hospital, and the requirement of informed consent was waived (institutional review board approval number: 2020-03-013). Detailed information on the KNHANES is available for reference on the website [24]. The KNHANES allows the public use of anonymous data once researchers sign and submit an agreement to use the data for research purposes only. The present analysis complies with the principles laid down in the amended Declaration of Helsinki.

\section{Sociodemographic factors}

The following sociodemographic factors were considered: age, gender, educational level, economic activity, marital status, and cohabitation status. Educational level was classified into four groups: elementary school graduates, middle school graduates, high school graduates, and university graduates. Marital status was divided into two groups: married and unmarried. Cohabitation status was divided into four groups: cohabiting, separated, bereaved, and divorced. Non-employment was divided into two groups: unemployed and non-economically active.

\section{Health behavior and medical factors}

Subjective health status and disease were investigated as medical factors. Subjective health status was classified into five groups: very good, good, average, bad, and very bad. The participants' usually perceived level of stress was investigated through the following statements:(1) "I feel a great deal of stress," (2) "I feel much stress," (3) "I feel a little stress," (4) "I hardly feel stress," (5) "Not applicable," and (6) "Unknown."

Choosing statement 1 or 2 indicated that participants experienced much stress, while choosing statement 3 or 4 indicated that they experienced less stress. A stress-sensitive participant was marked 1, and a less stress-sensitive participant was marked 0 . The average value was recorded as the stress recognition rate.

The EuroQol-5 Dimension (EQ-5D) is a comprehensive index used to assess health-related quality of life across 
five dimensions: exercise ability, self-management, daily activities, pain/discomfort, and anxiety/depression. Participants select one of three levels: "No problem at all," "Some problems," and "There are many problems" for the items on the five dimensions. A scoring conversion system was used to convert responses into a number between 1 (completely healthy) and -1 (not healthy at all) [25]. This was calculated using the estimated quality weight for the Korean population, which yielded the EQ-5D index. The following formula was used:

$$
\begin{aligned}
\mathrm{EQ}-5 \mathrm{D} \text { index } & =1-(0.0081+0.1140 * \mathrm{M} 2+0.6274 * \mathrm{M} 3 \\
& +0.0572 * \mathrm{SC} 2+0.2073 * \mathrm{SC} 3+0.0615 * \mathrm{UA} 2 \\
& +0.2812 * \mathrm{UA} 3+0.0581 * \mathrm{PD} 2+0.2353 * \mathrm{PD} 3 \\
& +0.0675 * \mathrm{AD} 2+0.2351 * \mathrm{AD} 3)
\end{aligned}
$$

Regarding smoking status, participants were classified as either smokers or non-smokers. People who smoked at the time of data collection or had smoked more than 100 cigarettes throughout their lives were defined as smokers. Alcohol consumption was investigated by calculating the amount of alcohol consumed by a participant per week in grams. The International Physical Activity Questionnaire was used to measure the extent of physical exercise; "regular exercise" was defined as exercising at least five times a week for $30 \mathrm{~min}$ per session or participation in vigorous physical activity three times a week for more than $20 \mathrm{~min}$ per session.

The participants' blood samples were randomly collected once after an 8-h fast and subjected to laboratory testing. The samples were immediately processed, refrigerated, and transported to the central laboratory (Neodin Medical Institute, Seoul, South Korea).

\section{Statistical analysis}

SPSS for Windows version 21.0 (IBM Corp., Armonk, NY, USA) was used for statistical analysis, and statistical significance was set at $p<0.05$. Owing to the complexity of KNHANES data, a complex sample analysis was conducted considering weights. Weights were applied according to the Centers for Disease Control and Prevention's guidelines for using KNHANES raw data.

For the overall results, a frequency analysis was performed using the Complex Samples Frequencies procedure. The complex sample Rao-Scott adjusted chi-square test and complex sample generalized linear model were used to compare differences in the general characteristics of patients with and without diabetes. A complex sample logistic regression test was used to analyze the factors associated with influenza vaccination among patients with diabetes.

\section{Results}

\section{General characteristics and diabetes prevalence (Table 1)}

There were two groups-patients with and without diabetes. Among patients with diabetes, there were more people with an educational level of primary school or lower than those who had a college degree or higher (36 vs. $15 \%$ ). Further, in this group, $18.3 \%$ were smokers, $33.4 \%$ engaged in aerobic exercise, and $44.3 \%$ drank monthly. The average stress perception rate was $23.5 \%, 96.6 \%$ were married, $72.1 \%$ were living with someone, $58.6 \%$ had private health insurance, and $16 \%$ performed limited physical activity. When evaluating their subjective health, the proportions of participants with diabetes who answered "very good" (1.4\%) and "good" (10.8\%) were low. Further, $46.9 \%$ were not employed and had lower EQ-5D scores than people not living with diabetes $(0.90 \pm 0.004$ vs. $0.96 \pm 0.001$; $p<0.0001$ ).

People with diabetes had higher influenza VCRs compared with those who did not have diabetes (60.6 vs. $34.1 \%$ ). People with diabetes were rarely inoculated twice a year and were more likely to be vaccinated in hospitals or clinics than in public health centers (62.8 vs. $34.9 \%$; $p<0.0001)$.

\section{Factors associated with influenza vaccination among people with diabetes (Table 2)}

A single analysis of factors associated with influenza vaccination among people with diabetes revealed that women had higher VCRs than men. Further, those with an educational level of primary school or lower had higher VCRs than those who had obtained a college degree or higher odds ratio [(OR) 1.928 (1.516-2.452), OR 4.215 (2.905-6.116), respectively; $p<0.001]$.

VCRs were higher among non-smokers, those who did not engage in aerobic exercise, and those who were not monthly drinkers (OR 2.437 (1.766-3.363), OR 1.339 (1.034-1.734), OR 2.180 (1.689-2.814), respectively; $p<0.001)$.

VCRs were low among unmarried participants and high among non-private health subscribers (OR 0.184 (0.081-0.418), OR 2.984 (2.323-3.835), respectively; $p<0.001)$. VCRs were also higher among those with limited physical activity and those who were not employed 
Table 1 General characteristics and diabetes prevalence

\begin{tabular}{|c|c|c|c|c|c|}
\hline & & $N=18,553$ & Normal & Diabetes & $p$ value \\
\hline \multirow[t]{2}{*}{ Gender } & Men & $8127(49.8)$ & 7240 (49.6) & $885(52.3)$ & \multirow[t]{2}{*}{0.058} \\
\hline & Women & $10,426(50.2)$ & $9496(50.4)$ & $924(47.7)$ & \\
\hline \multirow[t]{4}{*}{ Educational level } & $\leq$ Elementary school & $3656(13.9)$ & $2907(12.1)$ & $749(36)$ & \multirow[t]{4}{*}{$<0.0001$} \\
\hline & Middle school & $1782(8.3)$ & $1490(7.7)$ & $292(15.7)$ & \\
\hline & High school & $5617(33.2)$ & $5184(33.7)$ & $433(27.4)$ & \\
\hline & $\geq$ College & $6530(39.1)$ & $6296(41.1)$ & $234(15)$ & \\
\hline Smoking & & $3338(21.3)$ & 3044 (21.6) & $293(18.3)$ & $<0.0001$ \\
\hline Exercise & & $7554(43.8)$ & 7008 (44.6) & $546(33.4)$ & $<0.0001$ \\
\hline Drinking & & $9888(58.1)$ & $9158(59.2)$ & $729(44.3)$ & $<0.0001$ \\
\hline Stress recognition & & $4903(28.1)$ & $4503(28.5)$ & $398(23.5)$ & $<0.0001$ \\
\hline \multirow[t]{2}{*}{ Marital status } & Married & $15,477(76.8)$ & $13,708(75.1)$ & $1761(96.6)$ & \multirow[t]{2}{*}{$<0.0001$} \\
\hline & Single & $3076(23.2)$ & $3028(24.9)$ & $48(3.4)$ & \\
\hline \multirow[t]{4}{*}{ Cohabitation status } & Cohabiting & $12,837(65.6)$ & $11,560(65.1)$ & $1271(72.1)$ & \multirow[t]{4}{*}{$<0.0001$} \\
\hline & Separated & $116(0.6)$ & $100(0.5)$ & $16(0.9)$ & \\
\hline & Bereaved & $1705(6.6)$ & $1333(5.6)$ & $371(18.4)$ & \\
\hline & Divorced & $812(3.9)$ & $708(3.8)$ & $103(5.3)$ & \\
\hline Private health insurance & & $14,172(80.7)$ & $13,227(82.6)$ & 944 (58.6) & $<0.0001$ \\
\hline Activity limit & & $1514(6.5)$ & $1211(5.8)$ & $303(16)$ & $<0.0001$ \\
\hline \multirow[t]{5}{*}{ Subjective health } & Very good & $799(4.5)$ & $774(4.7)$ & $25(1.4)$ & \multirow[t]{5}{*}{$<0.0001$} \\
\hline & Good & $4164(23.9)$ & $3962(25)$ & $201(10.8)$ & \\
\hline & Average & 9203 (49.6) & $8402(50)$ & $801(45.5)$ & \\
\hline & $\mathrm{Bad}$ & $2769(14)$ & $2290(12.9)$ & 479 (26.7) & \\
\hline & Very bad & $765(3.1)$ & $9(16.1)$ & $216(10.7)$ & \\
\hline \multirow[t]{2}{*}{ Economic activity } & Employed & $10,677(61.1)$ & $9906(62.3)$ & $771(47.3)$ & \multirow[t]{2}{*}{$<0.0001$} \\
\hline & Non-employed & $6918(33.5)$ & $5980(32.4)$ & 938 (46.9) & \\
\hline \multirow[t]{2}{*}{ Influenza vaccination } & Yes & $7750(35.4)$ & $6557(33.3)$ & $1193(60.6)$ & \multirow[t]{2}{*}{$<0.0001$} \\
\hline & No & $9900(59.5)$ & $9373(61.6)$ & $527(34.1)$ & \\
\hline \multirow[t]{2}{*}{ Frequency of vaccination } & 1 & $7675(35.1)$ & $6493(33)$ & $1182(60.1)$ & \multirow[t]{2}{*}{$<0.0001$} \\
\hline & 2 & $75(0.3)$ & $64(0.3)$ & $11(0.5)$ & \\
\hline \multirow[t]{3}{*}{ Place of vaccination } & Public health center & $2239(24.5)$ & $1796(23)$ & 443 (34.9) & \multirow[t]{3}{*}{$<0.0001$} \\
\hline & Hospital, & $5210(70.4)$ & 4484 (71.6) & $726(62.8)$ & \\
\hline & Others & $295(5.0)$ & $272(5.4)$ & $23(2.2)$ & \\
\hline Age, years & & $40.87 \pm 0.27$ & $45.89 \pm 0.23$ & $63.60 \pm 0.36$ & $<0.0001$ \\
\hline EQ-5D & & $0.95 \pm 0.001$ & $0.96 \pm 0.001$ & $0.90 \pm 0.004$ & $<0.0001$ \\
\hline $\mathrm{HbA1c}$ & & $5.61 \pm 0.008$ & $5.51 \pm 0.01$ & $7.21 \pm 0.04$ & $<0.0001$ \\
\hline
\end{tabular}

Smoking: the percentage of people who had smoked more than five packets of cigarettes (100 cigarettes) in their lifetime or who smoked at the time of the survey; alcohol: percentage of participants who had an alcohol intake of more than once a month in the last year; non-employed: unemployed and non-economically active population; stress: percentage of stress in everyday life; EQ-5D: EuroQol-5 Dimension; HbA1c: hemoglobin A1c

Values are presented as number $(\%)$ or mean \pm standard deviation

${ }^{\mathrm{a}}$ The $p$ value was calculated using the complex sample Rao-Scott adjusted chi-square test and complex sample generalized linear model t-test
(OR 1.471 (1.048-2.065), OR 3.236 (2.473-4.235), respectively; $p<0.001)$.

The VCR increased as participants' age increased by 10 years, and as the EQ-5D score decreased by 1 (OR 2.586 (2.208-3.028), OR 10.939 (3.961-30.207), respectively; $p<0.001)$.
A multivariate analysis of the factors associated with influenza vaccination among people with diabetes demonstrated that VCRs were higher among those who were not employed, and that VCRs increased with age (OR 1.512 (1.087-2.105), OR 2.212 (1.822-2.686), respectively; $p<0.001)$. 
Table 2 Factors associated with influenza vaccination among people with diabetes

\begin{tabular}{llll}
\hline & & Univariate & Multivariate \\
& & OR $(95 \% \mathrm{CI})$ & OR (95\% CI $)$ \\
\hline Gender & Men & 1 & 1 \\
& Women & $1.928(1.516-2.452)$ & $1.095(0.79-1.519)$ \\
Educational level & $\leq$ Elementary & $4.215(2.905-6.116)$ & $1.266(0.785-2.042)$ \\
& Middle & $2.406(1.548-3.739)$ & $1.462(0.88-2.429)$ \\
& High & $1.409(0.947-2.096)$ & $1.225(0.766-1.961)$ \\
Smoking & $\geq$ College & 1 & 1 \\
Exercise & No & $2.437(1.766-3.363)$ & $1.319(0.902-1.930)$ \\
Drinking & No & $1.339(1.034-1.734)$ & $0.822(0.607-1.112)$ \\
Marital status & No & $2.180(1.689-2.814)$ & $1.134(0.826-1.558)$ \\
& Married & 1 & 1 \\
Private health insurance & Single & $0.184(0.081-0.418)$ & $0.566(0.193-1.659)$ \\
Activity limit & No & $2.984(2.323-3.835)$ & $1.061(0.774-1.456)$ \\
Economic activity & Yes & $1.471(1.048-2.065)$ & $0.821(0.531-1.271)$ \\
& Employed & 1 & 1 \\
Age & Non-employed & $3.236(2.473-4.235)$ & $1.512(1.087-2.105)$ \\
EQ-5D & $10-$ year increase & $2.586(2.208-3.028)$ & $2.212(1.822-2.686)$ \\
\hline
\end{tabular}

OR: odds ratio, CI: confidence interval.

Smoking: the percentage of people who had smoked more than five packets of cigarettes (100 cigarettes) in their lifetime or who smoked at the time of the survey; alcohol: percentage of participants who had an alcohol intake of more than once a month in the last year; non-employed: unemployed and non-economically active population; EQ-5D, EuroQol-5 Dimension

Adjusted for gender, age, educational level, smoking, exercise, drinking, marital status, private health insurance, activity limit, economic activity, age, EQ-5D score

${ }^{a}$ OR and $95 \%$ CI were calculated using a complex sample logistic regression test

\section{Discussion}

As previously discussed, people with diabetes are especially vulnerable to all infectious diseases, including influenza. Recently, people with diabetes were reported to have the second-highest mortality risk from COVID-19. Before highlighting the need for vaccination, however, it is necessary to evaluate the factors associated with the influenza VCR among people with diabetes. Previous South Korean studies that investigated the influenza VCR and associated factors have focused either on the general population $[26,27]$ or those with other chronic diseases [19, 20].

As people with diabetes are more susceptible to influenza and have a greater risk of medical complications from infection, the WHO and several National Immunization Technical Advisory Groups recommend annual influenza vaccination $[28,29]$.

A systematic review and meta-analysis of people with diabetes demonstrated that the effectiveness of the influenza vaccine differs slightly according to age [30]. Overall, the effectiveness of the influenza vaccine among working-age people (18-64 years) was 58\%. Although hospitalization due to influenza or pneumonia did not affect the overall mortality rate, it was found that vaccination for influenza decreased the hospitalization rate among people with diabetes of working age. In addition, vaccination for influenza among older adults (over 65 years old) has been demonstrated to reduce mortality rates from all causes, hospitalization rates from all causes, and hospitalization rates from influenza or pneumonia [30].

The findings mentioned above prove that the influenza vaccine is important, regardless of age and comorbid diseases. Thus, efforts to increase the influenza VCR are required. VCRs vary from country to country, and the factors affecting the rate within each country are different [31]. Therefore, in this analysis, we sought to identify the factors associated with the influenza VCR in South Korea.

According to the most recent data, the influenza VCR among people with diabetes in South Korea was $60.6 \%$. In a recent analysis, the goal was to increase the influenza VCR to $90 \%$ among people over 65 , and $60 \%$ among high-risk groups aged 18-64 in the USA [32]. In comparison with these numbers, the VCR in South Korea has met the target rate. The high VCR can be attributed to the South Korean medical system. In South Korea, the National Health Insurance Service is mandatory for everyone. Under this program, people receive deductions for a significant portion of medical costs. They also receive benefits when utilizing health 
management services. The cost of influenza vaccination varies slightly depending on the type of vaccine and hospital but does not exceed $\$ 50$. As per the national policy, people aged above 65 receive free immunization.

Although the VCR across risk groups and countries continues to increase, few countries are close to achieving the VCR target set by the WHO [28]. In the univariate analysis, many variables, such as gender, educational level, smoking, exercise, drinking, marital status, private health insurance, activity limit, economic activity, age, and EQ-5D score, were found to be associated with the VCR. However, the multivariate analysis revealed that only age and economic activity were associated with the VCR. Our findings demonstrated that there are only two individual patient factors (not employed and old age) that are associated with VCRs. In a previous Korean study of 32,268 individuals who participated in the KNHANES III-VI (2005-2015), the influenza VCR among diabetic patients was $50.0 \%$, which was lower than our results. For the non-diabetes group, the VCR was $38.2 \%$, which was higher than our results. Influenza VCR in diabetic patients was associated with socioeconomic (old age, female sex, high family income, medical assistance insurance) and health-related factors (dangerous drinking, obesity, lack of recent health screening) [7].

A study of 10 countries in Africa, Asia-Pacific, Eastern Europe, Latin America, and the Middle East demonstrated that the influenza VCR is not affected by patient factors [33]. This is similar to our findings and suggests that national programs are necessary to increase the influenza VCR [33]. Indeed, the UK has achieved its target VCR, and this is the result of active national support and health programs [34].

Although the rates were calculated in different years, a study conducted in five European countries demonstrated that the influenza VCRs among patients with chronic diseases, such as diabetes, were as follows: $59.4 \%$ in the UK, $29.8 \%$ in Germany, $36.7 \%$ in Italy, $34.4 \%$ in France, and $37.1 \%$ in Spain [35].

In a large-scale study in European countries conducted after the previously mentioned study, an increase in VCRs was observed [34]. However, they still did not reach the rate recommended by the WHO, indicating that management at the national level is necessary [34].

Contradictory to our results, some studies have suggested that several factors affect VCRs. A study in Singapore revealed that high income and high educational levels were associated with high VCR [36]. A study in Spain demonstrated that the following factors affect the influenza VCR: age, urban residence, income, marriage, health awareness, and caregivers [37]. A study of older adults in Brazil revealed that the factors that increase the influenza VCR include old age, being male, high income, high educational level, non-smoking, and solicitation [38]. In Canada, research demonstrated that higher education, higher income, smoking, increased levels of drinking, poor health perception, exercise, and city dwelling increased the influenza VCR [39]. A nationwide study in Spain revealed that old age, previous vaccination, chronic disease, and being female increased the influenza VCR among the vulnerable population [40]. In France, it was demonstrated that VCRs were higher in families with infants, higher educational levels, professional occupations, and previous influenza vaccination [40].

Influenza, which can be prevented through vaccination, causes significant economic losses. In the USA, the total annual cost related to influenza is $€ 10,000-17,000$ million [34]. French research estimates that the total cost related to influenza is over $€ 1796$ million per year [34]. These economic losses highlight the importance of increasing the influenza VCR.

Based on the most recent data, we analyzed the factors associated with the influenza VCR among people with diabetes in South Korea. However, it does have limitations. First, owing to the use of cross-sectional data, causality cannot be established. Second, not all factors that could be associated with vaccination history were considered. According to Korea's national policy, people over the age of 65 years are eligible for free vaccinations. Therefore, it is possible that the results of this analysis are biased. However, the reason why we included individuals aged 65 years and older and examined their employment status was to analyze the factors that affect the overall VCR in Korea, including the special policy background of Korea.

\section{Conclusions}

This study demonstrated that the influenza VCR is low in younger and employed people, possibly owing to time constraints. Overall, the influenza VCR is rarely associated with personal factors in people with diabetes. The results suggest that owing to the governmental healthcare system's major impact on the VCR and based on the fact that only two personal factors (not employed and old age) were associated with the influenza VCR, government intervention is necessary to increase the influenza VCR. Thus, it would be helpful to develop a national program that connects companies with public health centers with the joint goal of improving VCRs.

Acknowledgments The author would like to thank Editage (www.edita ge.co.kr) for English language editing.

Funding This work was supported by Wonkwang University (2022).

Data availability Not applicable.

Code availability Not applicable. 


\section{Declarations}

Ethics approval The study was approved by the Clinical Trial Screening Committee of Wonkwang University Hospital (approval number: 2020-03-013).

Consent to participate Not applicable.

Consent for publication Not applicable.

Conflicts of interest None.

\section{References}

1. Glezen WP, Greenberg SB, Atmar RL, Piedra PA, Couch RB. Impact of respiratory virus infections on persons with chronic underlying conditions. JAMA. 2000;283:499-505. https://doi.org/ 10.1001/jama.283.4.499.

2. Colquhoun AJ, Nicholson KG, Botha JL, Raymond NT. Effectiveness of influenza vaccine in reducing hospital admissions in people with diabetes. Epidemiol Infect. 1997;119:335-41. https:// doi.org/10.1017/s095026889700825x.

3. Wang IK, Lin CL, Chang YC, Lin PC, Liang CC, Liu YL, et al. Effectiveness of influenza vaccination in elderly diabetic patients: a retrospective cohort study. Vaccine. 2013;31:718-24. https://doi. org/10.1016/j.vaccine.2012.11.017.

4. Lau D, Eurich DT, Majumdar SR, Katz A, Johnson JA. Effectiveness of influenza vaccination in working-age adults with diabetes: a population-based cohort study. Thorax. 2013;68:658-63. https:// doi.org/10.1136/thoraxjnl-2012-203109.

5. Li S, Wang J, Zhang B, Li X, Liu Y. Diabetes mellitus and causespecific mortality: a population-based study. Diabetes Metab J. 2019;43:319-41. https://doi.org/10.4093/dmj.2018.0060.

6. Dos Santos G, Tahrat H, Bekkat-Berkani R. Immunogenicity, safety, and effectiveness of seasonal influenza vaccination in patients with diabetes mellitus: a systematic review. Hum Vaccin Immunother. 2018;14:1853-66. https://doi.org/10.1080/21645 515.2018.1446719.

7. Shin HY, Chung JH, Hwang HJ, Kim TH. Factors influencing on influenza vaccination and its trends of coverage in patients with diabetes in Korea: a population-based cross-sectional study. Vaccine. 2018;36:91-7. https://doi.org/10.1016/j.vaccine.2017. 11.035 .

8. Diepersloot RJ, Bouter KP, Hoekstra JB. Influenza infection and diabetes mellitus: case for annual vaccination. Diabetes Care. 1990;13:876-82. https://doi.org/10.2337/diacare.13.8.876.

9. Diepersloot RJ, Bouter KP, Beyer WE, Hoekstra JB, Masurel N. Humoral immune response and delayed type hypersensitivity to influenza vaccine in patients with diabetes mellitus. Diabetologia. 1987;30:397-401. https://doi.org/10.1007/BF00292541.

10. Carrillo-Santisteve P, Ciancio BC, Nicoll A, Luigi LP. The importance of influenza prevention for public health. Hum Vaccin Immunother. 2012;8:89-95. https://doi.org/10.4161/hv.8.1.19066.

11. Jiménez-Garcia R, Lopez-de-Andres A, Hernandez-Barrera V, Gómez-Campelo P, San Andrés-Rebollo FJ, de Burgos-Lunar $\mathrm{C}$, et al. Influenza vaccination in people with type 2 diabetes, coverage, predictors of uptake, and perceptions. Result of the MADIABETES cohort a 7 years follow up study. Vaccine. 2017;35:101-8. https://doi.org/10.1016/j.vaccine.2016.11.039.

12. Yang L, Nan H, Liang J, Chan YH, Chan L, Sum RW, et al. Influenza vaccination in older people with diabetes and their household contacts. Vaccine. 2017;35:889-96. https://doi.org/ 10.1016/j.vaccine.2017.01.004.

13. Villarroel MA, Vahratian A. Vaccination coverage among adults with diagnosed diabetes, United States, 2015. NCHS Data Brief. 2016;265:1-8.

14. Cheng LJ, Chen JH, Lin MY, Chen LC, Lao CH, Luh H, et al. A competing risk analysis of sequential complication development in Asian type 2 diabetes mellitus patients. Sci Rep. 2015;5:15687. https://doi.org/10.1038/srep15687.

15. Critchley JA, Carey IM, Harris T, DeWilde S, Hosking FJ, Cook DG. Glycemic control and risk of infections among people with type 1 or type 2 diabetes in a large primary care cohort study. Diabetes Care. 2018;41:2127-35. https://doi.org/10.2337/ dc18-0287.

16. Breitling LP. Evidence of non-linearity in the association of glycemic control with influenza/pneumonia mortality: a study of 19 000 adults from the US general population. Diabetes Metab Res Rev. 2016;32:111-20. https://doi.org/10.1002/dmrr.2681.

17. Yang J, Yan H, Feng LZ, Yu HJ. Cost-effectiveness of potential government fully-funded influenza vaccination in population with diabetes in China. Zhonghua Yu Fang Yi Xue Za Zhi [Chinese J Prev Med]. 2019;53:1000-6. https://doi.org/10.3760/cma.j.issn. 0253-9624.2019.10.009.

18. Shin HY, Hwang HJ, Chung JH. Factors influencing influenza vaccination among patients with chronic obstructive pulmonary disease: a population-based cross-sectional study. Asia Pac J Public Health. 2017;29:560-8. https://doi.org/10.1177/1010539517 735415.

19. Kee SY, Cheong HJ, Chun BC, Kim WJ. Influenza vaccination coverage rate and factors associated with vaccination in people with chronic disease. Infect Chemother. 2011;43:406-11. https:// doi.org/10.3947/ic.2011.43.5.406.

20. Cho HK, Jeong JS, Moon S, Kim MN. Current immunization status and factors affecting the influenza vaccination in kidney transplant patients. J Korean Biol Nurs Sci. 2016;18:118. https:// doi.org/10.7586/jkbns.2016.18.2.118.

21. Lim J, Eom CS, Kim KH, Kim S, Cho B. Coverage of influenza vaccination among elderly in South Korea: a population based cross sectional analysis of the season 2004-2005. J Korean Geriatrics Soc. 2009;13:215-21.

22. Kwon DS, Kim K, Park SM. Factors associated with influenza vaccination coverage among the elderly in South Korea: The Fourth Korean National Health and Nutrition Examination Survey (KNHANES IV). BMJ Open. 2016;6:e012618. https://doi.org/10. 1136/bmjopen-2016-012618.

23. Ryu SY, Kim SH, Park HS, Park J. Influenza vaccination among adults 65 years or older: a 2009-2010 community health survey in the Honam region of Korea. Int J Environ Res Public Health. 2011;8:4197-206. https://doi.org/10.3390/ijerph8114197.

24. Kweon S, Kim Y, Jang MJ, Kim Y, Kim K, Choi S, et al. Data resource profile: The Korea national health and nutrition examination survey (KNHANES). Int J Epidemiol. 2014;43:69-77. https:// doi.org/10.1093/ije/dyt228.

25. Balestroni G, Bertolotti G. EuroQol-5D (EQ-5D): an instrument for measuring quality of life. Monaldi Arch Chest Dis. 2012;78:155-9. https://doi.org/10.4081/monaldi.2012.121.

26. Yang HJ, Cho S-I. Influenza vaccination coverage among adults in Korea: 2008-2009 to 2011-2012 seasons. Int J Environ Res Public Health. 2014;11:12162-73. https://doi.org/10.3390/ijerp h111212162.

27. Byeon GR, Hur YR, Kang JH, Park HA, Kim KW, Cho YG, et al. Influenza vaccination status in Korean adult population in relation with socioeconomic and medical factors. Korean J Health Promot. 2016;16:20-31. 
28. World Health Organization. Meeting of the Strategic Advisory Group of Experts on Immunization, April 2012-conclusions and recommendations. Wkly Epidemiol Rec. 2012;87:201-16.

29. Centers for Disease Control and Prevention. Prevention and control of seasonal influenza with vaccines. Recommendations of the Advisory Committee on Immunization Practices-United States, 2013-2014. MMWR Recomm Rep. 2013;62:906.

30. Remschmidt C, Wichmann O, Harder T. Vaccines for the prevention of seasonal influenza in patients with diabetes: systematic review and meta-analysis. BMC Med. 2015;13:53. https://doi.org/ 10.1186/s12916-015-0295-6.

31. Lewis-Parmar H, McCann R. Achieving national influenza vaccine targets-an investigation of the factors affecting influenza vaccine uptake in older people and people with diabetes. Commun Dis Public Health. 2002;5:119-26.

32. Takayama M, Wetmore CM, Mokdad AH. Characteristics associated with the uptake of influenza vaccination among adults in the United States. Prev Med. 2012;54:358-62. https://doi.org/10. 1016/j.ypmed.2012.03.008.

33. de Lataillade C, Auvergne S, Delannoy I. 2005 and 2006 seasonal influenza vaccination coverage rates in 10 countries in Africa, Asia Pacific, Europe, Latin America, and the Middle East. J Public Health Policy. 2009;30:83-101. https://doi.org/10.1057/jphp. 2008.40.

34. Palache A, Oriol-Mathieu V, Fino M, Xydia-Charmanta M. Seasonal influenza vaccine dose distribution in 195 countries (20042013): little progress in estimated global vaccination coverage. Vaccine. 2015;33:5598-605. https://doi.org/10.1016/j.vaccine. 2015.08.082.
35. Blank PR, Schwenkglenks M, Szucs TD. Influenza vaccination coverage rates in five European countries during season 2006/07 and trends over six consecutive seasons. BMC Public Health. 2008;8:272. https://doi.org/10.1186/1471-2458-8-272.

36. Tan EK, Lim LH, Teoh YL, Ong G, Bock HL. Influenza and seasonal influenza vaccination among diabetics in Singapore: knowledge, attitudes, and practices. Singapore Med J. 2010;51:623.

37. Nagata JM, Hernández-Ramos I, Kurup AS, Albrecht D, VivasTorrealba C, Franco-Paredes C. Social determinants of health and seasonal influenza vaccination in adults $\geq 65$ years: a systematic review of qualitative and quantitative data. BMC Public Health. 2013;13:388. https://doi.org/10.1186/1471-2458-13-388.

38. Dip RM, Cabrera MA. Influenza vaccination in non-institutionalized elderly: a population-based study in a medium-sized city in Southern Brazil. Cad Saude Publica. 2010;26:1035-44. https:// doi.org/10.1590/S0102-311X2010000500025.

39. Andrew MK, McNeil S, Merry H, Rockwood K. Rates of influenza vaccination in older adults and factors associated with vaccine use: a secondary analysis of the Canadian Study of Health and Aging. BMC Public Health. 2004;4:36. https://doi.org/10. 1186/1471-2458-4-36.

40. Vaux S, Van Cauteren D, Guthmann JP, Le Strat Y, Vaillant V, de Valk $\mathrm{H}$, et al. Influenza vaccination coverage against seasonal and pandemic influenza and their determinants in France: a crosssectional survey. BMC Public Health. 2011;11:30. https://doi.org/ 10.1186/1471-2458-11-30.

Publisher's note Springer Nature remains neutral with regard to jurisdictional claims in published maps and institutional affiliations. 